\title{
Targets of jokes (Satirical Shows)
}

\section{AUTHOR}

Dennis Lichtenstein, Cordula Nitsch

\section{KEYWORDS}

satire, jokes, satirical show, targets of jokes, political actors, humor

\section{BRIEF DESCRIPTION}

With the variable "targets of jokes" it is analyzed which actors are evaluated in satirical shows (either by criticism or as a target of a joke). Targets of jokes can be celebrities and societal actors, however, the greatest attention is given to political actors. Political actors can either be collective actors such as parties and other political organizations or individual politicians. Due to cultural specifics in the U.S., the coding of political actors as targets of jokes is often expanded to the family and staff of presidential candidates and other high-ranking politicians in American studies (Lichter et al., 2015).

\section{FIELD OF APPLICATION/THEORETICAL FOUNDATION}

The variable is used to analyze balance in jokes (e.g., distribution of jokes on political actors) and is an indicator for political plurality.

\section{REFERENCES/COMBINATION WITH OTHER METHODS OF DATA COLLECTION}

The variable is used in content analysis that have been combined with experimental studies that analyze priming effects (Matthes \& Rauchfleisch, 2013)

\section{EXAMPLE STUDY:}

Nitsch \& Lichtenstein (2013)
INFORMATION ON NITSCH \& LICHTENSTEIN, 2013

Authors: Cordula Nitsch, Dennis Lichtenstein Research question/ research interest: Depiction of politics (topics and actors) in satirical shows vs. news shows

Object of analysis: Satirical Show (Harald Schmidt Show), news show (Tagesschau)

Timeframe of analysis: $2009-2010$

\section{INFORMATION ABOUT VARIABLE}

Variable name/definition: Akteure [actors]

Gewertet werden nur politisch relevante Akteure, die in der Sendung angesprochen werden oder selbst sprechen. Ein Akteur gilt als politischer Akteur, wenn es sich dabei entweder um eine politische Institution (z.B. EU, Nato, UNO, Parlament) oder um einen demokratisch legitimierten Individualakteur (z.B. Bundespräsident, Politiker unterschiedlicher Parteien) handelt. Pro Thema wird jeder Akteur nur einmal codiert. Enthält ein Thema keinen Akteur, so wird die Variable nicht ausgefüllt.

[Only politically relevant actors who are addressed in the satirical shows or who speak on their own behalf are considered. An actor is considered a political actor if it is either a political institution (e.g. EU, NATO, UN, parliament) or a democratically legitimated individual actor (e.g. Federal President, politicians from different parties). If a topic contains no actor, the variable is not coded.]

Level of analysis: Beitragsebene

Scale level: Nominal

Reliability: Identifikation der Akteure: .82, Akteursvariablen: .81 see Table 1 


\section{REFERENCES}

Nitsch, C. \& Lichtenstein, D. (2013). Politik mal anders: Die Politikdarstellung in „Harald Schmidt“ im Kontrast zur „Tagesschau“ [A

different serving of politics: the depiction of politics in Harald Schmidt as opposed to Tagesschau] Publizistik, 58(4), 389-407.

\section{Table 1.}

\begin{tabular}{|c|c|c|c|c|c|}
\hline D national & D regional & $\mathbf{E U}$ & Ausland & & Bereich \\
\hline \multirow[t]{12}{*}{$1 \mathrm{xxx}$} & \multirow[t]{12}{*}{$2 \mathrm{xxx}$} & \multirow[t]{12}{*}{$3 x x x$} & \multirow[t]{12}{*}{$4 x x x$} & 100 & $\begin{array}{l}\text { Politiker } \\
\text { Land/EU als Ganzes } \\
\text { z.B. „die USA haben entschieden“, } \\
\text { "Griechenland ist pleite“, „die EU } \\
\text { sagt..." }\end{array}$ \\
\hline & & & & 110 & $\begin{array}{l}\text { Präsident } \\
\text { z.B. Bundespräsident }\end{array}$ \\
\hline & & & & 111 & $\begin{array}{l}\text { Regierungschef } \\
\text { z.B. Bundeskanzler }\end{array}$ \\
\hline & & & & 112 & $\begin{array}{l}\text { Regierung } \\
\text { Als Gesamtheit oder einzelne Minister } \\
\text { auf nationaler Ebene; für EU: Kom- } \\
\text { mission und Rat }\end{array}$ \\
\hline & & & & 113 & $\begin{array}{l}\text { Die Opposition } \\
\text { Nur als Gesamtheit, auf nationaler } \\
\text { Ebene }\end{array}$ \\
\hline & & & & 114 & Parlament \\
\hline & & & & 115 & $\begin{array}{l}\text { Partei } \\
\text { z.B. „die SPD hat sich mit der CDU } \\
\text { darauf geeinigt..." }\end{array}$ \\
\hline & & & & 116 & $\begin{array}{l}\text { Spitzenfunktionär in Partei } \\
\text { z.B. Parteichef, Generalsekretär }\end{array}$ \\
\hline & & & & 117 & $\begin{array}{l}\text { Sonstiges Parteimitglied/Parlamenta- } \\
\text { rier }\end{array}$ \\
\hline & & & & 118 & Ehemaliger Politiker \\
\hline & & & & 119 & Ehemalige Regierung \\
\hline & & & & 199 & $\begin{array}{l}\text { Politischer Akteur, nicht näher zuzu- } \\
\text { ordnen }\end{array}$ \\
\hline
\end{tabular}

\section{Weitere Akteure:}

5101 Nato

5102 UNI

5103G8-Staaten

5999Anderer internationaler politischer Akteur 